\title{
Effect of Hematocrit Level on the Blood Flow through Stenosed Artery: A Theoretical Study
}

\author{
A. Malek ${ }^{\text {* }}$, A. Hoque ${ }^{2}$, Md. Mohiuddin ${ }^{3}$ \\ 1,2 Department of Mathematics, University of Rajshahi, Rajshahi-6205, BANGLADESH \\ ${ }^{3}$ Department of Natural Sciences, Daffodil International University, BANGLADESH \\ "Corresponding Contact: \\ Email: malekbio@gmail.com
}

\begin{abstract}
In the paper we have focused the effect of Hematocrit on flow parameters, such as resistance, frictional resistance, and skin friction due to stenosis in the artery taking blood as non-Newtonian fluid. It has been found that resistance increases with the increasing of hematocrit level and stenosis height. It is also shown that a skin-friction increase with increasing of stenosis height and decreases with the decreasing of stenosis height. Moreover the effect of stenosis shape parameter is observed on resistance and skin-friction due to different hematocrit level. Finally we compare our result graphically.
\end{abstract}

Key words

Hematocrit; Resistance; Skin-friction; Stenosis height; Shape parameter

\section{INTRODUCTION}

Atherosclerosis is the most leading causes of the death due to heart diseases and the first symptom of atherosclerotic cardiovascular disease of men and women is heart attack, so it is very impotent to study the blood flow through the stenosed arteries. Stenosis causes on the arterial wall due to the accumulation of cholesterols and fats and the abnormal growth of tissue and obstructs the blood flow easily, although the exact mechanism of the formation of stenosis is not fully understood. The buildup that results is called plaque which might be the cause of partially or totally blockage of arterial blood flow. Mathematical modeling (analytical and computational) can be used for arterial flow and which is non-invasive method of studying (Wong et. al., 2010).

The behavior of arterial blood flow in the human body depends on the limiting value of shear rate and it varies from zero to approximately $1000 \mathrm{~s}^{-1}$ in several large arteries over a cardiac cycle (Cho and Kensey, 1991).Taylor (1959) observed typically a non-Newtonian pulsatile flow exists at low shear rate and blood exhibits Newtonian characteristic at high shear rate in the large arteries like aorta.Low shear rate is observed in some regions such as bifurcations, stenosis and aneurysms and blood flow through stenised artery where the flow behaves non Newtonian characteristics (Leondes, 2000). But shear rate is a function of blood composition which is dominated by hematocrit (Crowley and Pizziconi, 2005) and hematocrit can influence 
the blood viscosity or shear rate (Eckmann, 2000;Paut and Bissonnette, 2002), so hematocrit plays important properties of blood flow on stenosis. Ting and $\mathrm{Wu}$ (2006) investigated the effect of non-Newtonian properties on the shear stress distribution acting on the surface under the physiological conditions and reported that hematocrits effects on non-Newtonian properties. Wall shear stress and effective viscosity are found to be higher due to the influence of Hematocrit under the investigation of blood flow through a tapered artery in the presence of mild stenosis (Biswas and Chakraborty, 2010).

Some researchers (Wong et al., 2010; Blair and Spanner, 1974; Misra and Shit, 2006; Sing and Sing, 2012;Sriyab, 2014, etc.) focused that under certain conditions blood behaves like a power law fluid, Casson's fluid and Herschel-Bulkley's fluid. Wong et al., (2010) took power law fluid model as blood flow through the stenosed arteries and examined that the variability in the arterial wall geometry effects on the blood flow resistance. Halder (1985) found the maximum resistance to flow at throat of the symmetric stenosis by considering the power law fluid. Blood behaves likes a Casson's fluid in the case of moderate shear rate and that is not much of difference between Casson's and Herschel-Bulkley's fluid (Blair and Spanner, 1974). The Herschel-Bulkley's model is of general type and results obtained by this model to derive the corresponding Newtonian fluid, Bingham fluid and also power law fluid by putting specific results for values for the parameters (Misra and Shit, 2006). They considered Herschel-Bulkley to represent the non-Newtonian character of blood and noticed that the resistance of flow and skin-friction increase as the stenosis height increases. Pralhad and Schultz (2004) observed that the values of shear stress increase with the increasing of stenosis height and decrease with the increasing of couple stress parameters. Recently, Misra and Shit (2007), Biswas and Chakraborty (2009) have developed mathematical models for blood flow through stenosed arterial segment, by taking a velocity slip condition at the constricted wall.Sing and Sing (2012) studied the blood flow through radially non-symmetric stenosed artery and observed that resistance to flow increases as stenosis height or yield stress increases and decrease as stenosis shape increases and they quite hematocrit effect in blood flow modeling. Sriyab (2014) investigated the resistance and skin friction by K-L method in stenosis artery in presence of non-Newtonian blood with the Casson model which showed that skin friction increases with the increasing of stenosis length but did not investigate the effect of hematocrit level.

From the above discussion, it is clear that hematocrit plays important role in the blood system. So, the objective of this study to investigate the effects of hematocrit in the blood flow system, especially on resistance and skin friction through the stenosed artery.

\section{Mathematical Formulation}

To analysis the non-Newtonian blood flow in the stenosed arteries, various mathematical models were developed by Forrester and Young (1970), Misra and Shit (2007), Biswas and Chakraborty (2009) etc. But they did not determine the hemotocrit using the above model that we want to calculate it. We assume that the vessel is straight and symmetric and the flow is steady, laminar and fully developed to investigate the flow properties of nonNewtonian fluid through stenosed artery. The constitutive equation in the one dimensional flow in an artery is of the form (Young, 1968):

$\frac{d p}{d z}+\frac{1}{r} \frac{d(r \tau)}{d r}=0$

where $p$ and $\tau$ represent the presser at any point and shear stress of blood respectively. 
The non-Newtonian behavior of blood depends on the interaction of the red blood cells (hematocrit) and hematocrit plays viscosity of blood and shear rate. The constitutive equation of blood to analysis at the significant level of hematocrit on the shear rate modeled by Wlburn-Schneck (1976) as follows:

$$
\tau=a_{1} \exp \left(a_{2} H+\frac{a_{3}}{H^{2}}\right) \dot{\gamma}^{\left(1-a_{4} H\right)}
$$

where $\dot{\gamma}$ and $H$ are the shear strain rate and hematocrit level on the blood, and $a_{1}, a_{2}, a_{3}$, and $a_{4}$, are constant corresponding to $0.000797,0.0608,377.7515$ and 0.00499 for whole blood analysis and $\dot{\gamma}=-(d u / d r) u$ is the axial velocity of blood. In the empirical constitutive Eq. (2), hematocrit is an independent variable and shear rate (non-Newtonian properties) can be investigated from different level of hematocrit. So, the above equations can be solved using flowing boundary conditions:

$$
\begin{aligned}
& u=0 \text { at } r=R(z), \text { no }- \text { slip condition } \\
& \tau \text { is finite at } r=0, \quad \text { regularity condition }
\end{aligned}
$$

Shear rate or viscosity of the blood depends on the variability of stenosis geometry. We consider an axially symmetric and radially non-symmetric stenosis and the variation of the radius of the stenosed artery portion of the can be written as following (Singh and Singh, 2012):

$\frac{R}{R_{0}}=\left\{\begin{array}{cl}1-\frac{\delta}{R_{0}} \frac{s^{(s-1)}}{l^{s}(s-1)}\left[l^{s-1}(z-d)-(z-d)^{s}\right] & d \leq z \leq d+l \\ 1 & \text { otherwise }\end{array}\right.$

where $R_{0}$ and Rare the radius of the artery segment outside the stenosis and stenosis portion of the artery segment, $\delta$ is maximum height of the stenosis which is constant smaller then $R_{0}$ and $l$ are and length of the stenosis respectively. $s \geq 2$ is the shape parameter of the stenosis and variation of $s$ forms the profile given in the Fig. 1 and variation of shape parameter can be studied for changing the value of $s$. dis the location of the stenosis.

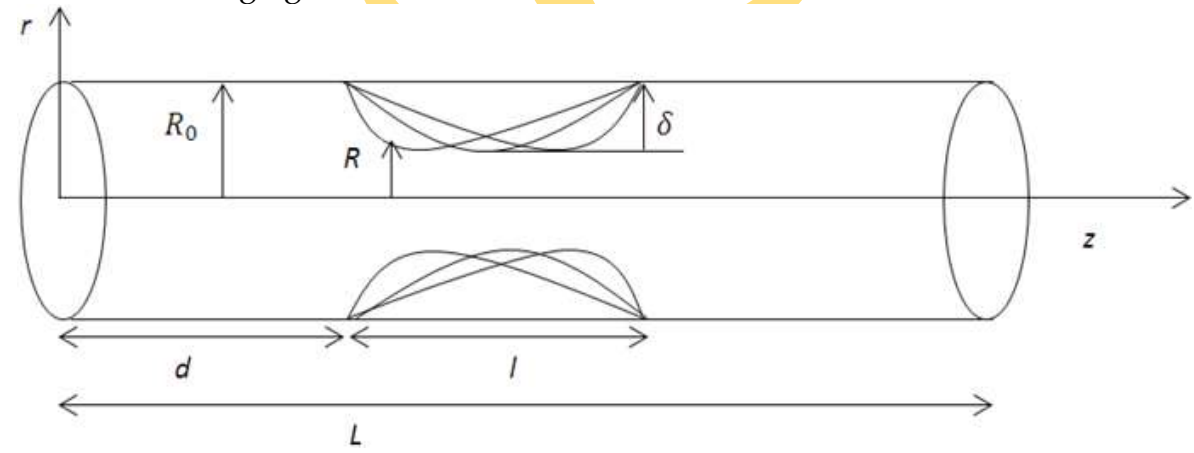

Fig.1: Geometry of the arterial segment with stenosis

\section{SOLUTION}

The constitutive Eq. (2) may be written as

$-\frac{d u}{d r}=f(\tau)=\left(\frac{1}{a_{1}}\right)^{\frac{1}{1-a_{4} H}} e^{-\frac{a_{2} H^{3}+a_{3}}{H^{2}-a_{4} H^{3}}(\tau)^{\frac{1}{1-a_{4} H}}}$

Integrating Eq. (1) and using the boundary condition (regularity condition), we get the shear stress and skin-friction as 
$\tau=-\frac{r}{2} \frac{d p}{d z}$ and $\tau_{R}=-\frac{R}{2} \frac{d p}{d z}$.

The volumetric flow rate $Q$ is given by the Rabinowitsch equation

$Q=\frac{\pi R^{3}}{\tau_{R}^{3}} \int_{0}^{\tau_{R}} \tau^{2} f(\tau) d \tau$.

Substituting Eq. (4) into Eq. (6), we obtain

$Q=\frac{\pi R^{3}\left(1-a_{4} H\right)}{4-3 a_{4} H}\left(\frac{1}{a_{1}}\right)^{\frac{1}{1-a_{4} H}} e^{-\frac{a_{2} H^{3}+a_{3}}{H^{2}-a_{4} H^{3}} \tau_{R}^{\frac{1}{1-a_{4} H}} .}$

Using Eq. (5) into Eq. (7), we have

$\frac{d p}{d z}=-2\left[\frac{Q\left(4-3 a_{4} H\right)}{\pi\left(1-a_{4} H\right)}\right]^{\left(1-a_{4} H\right)} e^{\frac{a_{2} H^{3}+a_{3}}{H^{2}}} a_{1} R^{-\left(4-3 a_{4} H\right)}$.

IntegratingEq. (8) taking the limits $p=p_{1}$ at $z=0$ and $p=p_{2}$ at $z=L$, we get

$$
p_{2}-p_{1}=2\left[\frac{Q\left(4-3 a_{4} H\right)}{\pi\left(1-a_{4} H\right)}\right]^{\left(1-a_{4} H\right)} e^{\frac{a_{2} H^{3}+a_{3}}{H^{2}}} a_{1} R_{0}^{-\left(4-3 a_{4} H\right)} \int_{0}^{L}\left(\frac{R}{R_{0}}\right)^{-\left(4-3 a_{4} H\right)} d z \ldots
$$

Thus the resistance to flow $\lambda$ defined by SomchaiSriyab (2014) can be written as

$\lambda=\frac{p_{2}-p_{1}}{Q}$

The above equation becomes with the help of Eq. (9) as

$\lambda=T \int_{0}^{L}\left(\frac{R}{R_{0}}\right)^{-\left(4-3 a_{4} H\right)} d z$

where

$T=2\left[\frac{4-3 a_{4} H}{\pi\left(1-a_{4} H\right)}\right]^{\left(1-a_{4} H\right)} e^{\frac{a_{2} H^{3}+a_{3}}{H^{2}}} a_{1} \frac{1}{Q^{a_{4} H}} R_{0}^{-\left(4-3 a_{4} H\right)}$.

Thus, Eq. (1) can be written as

$\lambda=T\left(\int_{0}^{d}\left(\frac{R}{R_{0}}\right)^{-\left(4-3 a_{4} H\right)} d z+\int_{d}^{d+l}\left(\frac{R}{R_{0}}\right)^{-\left(4-3 a_{4} H\right)} d z+\int_{d+l}^{L}\left(\frac{R}{R_{0}}\right)^{-\left(4-3 a_{4} H\right)} d z\right)$

or, $\quad \lambda=T(I+L-l)$

where

$I=\int_{d}^{d+l}\left(\frac{R}{R_{0}}\right)^{-\left(4-3 a_{4} H\right)} d z$ and $\mathrm{L}$ and $l$ are the length of vessels and stenosis as described in the Fig.1.

If there is no stenosis, the resistance to flow $\lambda_{N}$ is given by

$\lambda_{N}=T L$.

In dimensionless form, the flow resistance to flow may be expressed as

$\bar{\lambda}=\frac{\lambda}{\lambda_{N}}=1-\frac{l}{L}+\frac{I}{L}$. 
Substituting the expression for $\frac{R}{R_{0}}$ from Eq.(3), the integral I reduce to

$I=\int_{d}^{d+l}\left(1-\frac{\delta}{R_{0}} \frac{s^{(s-1)}}{l^{s}(s-1)}\left[l^{s-1}(z-d)-(z-d)^{s}\right]\right)^{-\left(4-3 a_{4} H\right)} d z$.

The skin-friction is obtained

$\tau_{R}=-\frac{R}{2} \frac{d p}{d z}=\left[\frac{Q\left(4-3 a_{4} H\right)}{\pi\left(1-a_{4} H\right)}\right]^{\left(1-a_{4} H\right)} e^{\frac{a_{2} H^{3}+a_{3}}{H^{2}}} a_{1} R^{-3\left(1-a_{4} H\right) .}$

In the absence of any constriction $\left(R=R_{0}\right)$ the skin-friction can be expressed as

$\tau_{N}=\left[\frac{Q\left(4-3 a_{4} H\right)}{\pi\left(1-a_{4} H\right)}\right]^{\left(1-a_{4} H\right)} e^{\frac{a_{2} H^{3}+a_{3}}{H^{2}}} a_{1} R_{0}^{-3\left(1-a_{4} H\right) .}$

which is the skin-friction of normal artery.

In dimensionless form, the skin-friction can be expressed as

$\bar{\tau}=\frac{\tau_{R}}{\tau_{N}}=\left(\frac{R}{R_{0}}\right)^{-3\left(1-a_{4} H\right)}$

$=\left(1-\frac{\delta}{R_{0}} \frac{s^{(s-1)}}{l^{s}(s-1)}\left[l^{s-1}(z-d)-(z-d)^{s}\right]\right)^{-3\left(1-a_{4} H\right)} d \leq z \leq d+l$

\section{RESULTS AND Discussion}

The microcirculation is the site of most of resistance to flow and skin-friction in stenosed artery, which depends on the architecture of the micro vascular network and on the rheological behavior of blood flowing through it. The effect of hematocrit on flow parameters (resistance, frictional resistance and skin friction) due to stenosis in the artery for whole blood has been studied analytically. For presenting the result graphically, the values of the parameters are consider with its range as $\mathrm{H}=20 \%-50 \%, \mathrm{Q}=1, \mathrm{~L}=1, \mathrm{l}=.5, \mathrm{~d}=.25, \mathrm{R}_{0}=.5, \delta / \mathrm{R}=0-0.5$.

Resistance of blood through the blood vessels influences cardiovascular function. To obtain the resistance of blood flow through the stenosed artery, taking blood as Non-Newtonian fluid, we have used Wlburn-Schneck (1976) model for whole blood and get the resistance of blood represented by Eq. (11) analytically. In graphically representation of the resistance given Eq.(11) the effect of hematocrit on the resistance observing the hematocrit level at $\mathrm{H}=50 \%, 40 \%$, $30 \%, 20 \%$ with the variation of the stenosis height have been shown in Fig. (1) and shape parameter $s=2$. In this Fig. (1) we have also shown that the effect of stenosis height considering the resistance at vertical axis and stenosis height at horizontal axis for different level of hematocrit. Graphically the result shows that resistance increases with the increasing of hematocrit level and stenosis height. In Fig.(2),we have also presented the effect of shape parameter which shows that for increasing of shape parameter resistance increases. We have also observed from the figure that the effect of $s=2,2.25,2.5$ and 2.75 for different shaped profile of stenosis due to increase of stenosis height for hematocrit level at $\mathrm{H}=50 \%$.

The expression of skin friction and the dimensionless form of skin friction with the effect of stenosis in the artery have been expressed by Eq.(14) and Eq.(16) analytically. The graphical representation of skin friction $(\bar{\tau})$ along the vertical axis taking $\mathrm{z}$ as horizontal axis for different values of stenosis height $\delta / R_{0}=0.5,0.4,0.3$, and 0.2 is plotted in Fig. (5). It is seen that the skinfriction $(\bar{\tau})$ increases significantly with the increasing of stenosis height. In Fig.(6) the variation 
of skin friction has been represented along the $\mathrm{z}$ axis of the artery taking shape parameter $\mathrm{s}=2$ and stenosis height $\delta / R_{0}=0.5$ for different levels of hematocrit. It is observed that at the initial of the stenosis skin friction is same for different level of hematocrit but it decreases with the increasing of hematocrit level and it is highly indicated at the middle of the stenosis. The skinfriction has also been sketched for different values of shape parameter $s=2,2.25,2.5$ and 2.75 for hematocrit level at $\mathrm{H}=50 \%$ which is shown in Fig. (7).

Finally we have compared our result using Hematocrit $\mathrm{H}=50 \%$ and shape parameter $\mathrm{s}=2.5$ with singh's (2010) result and Misra's (2006) result in Fig. (8). B. Singh (2010) and Misra(2006) taken power law fluid Casson fluid $(n=2 / 3)$ respectively but we take Wlburn-Schneck model for the non-Newtonian behavior of blood and we also take different stenosis model.

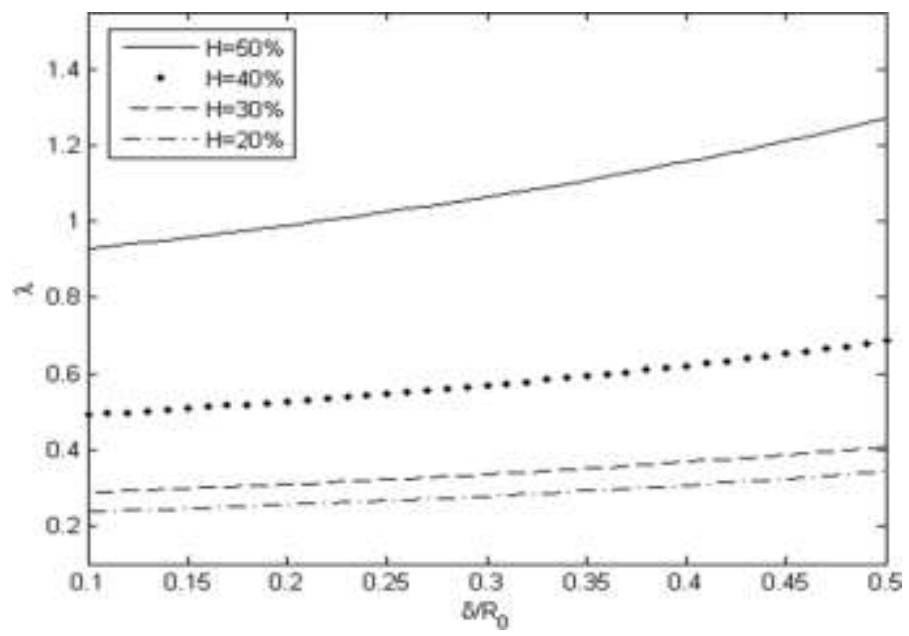

Fig 1: Variation of resistance of flow along the stenosis height for different value of hematocrit level $\mathrm{H}$.

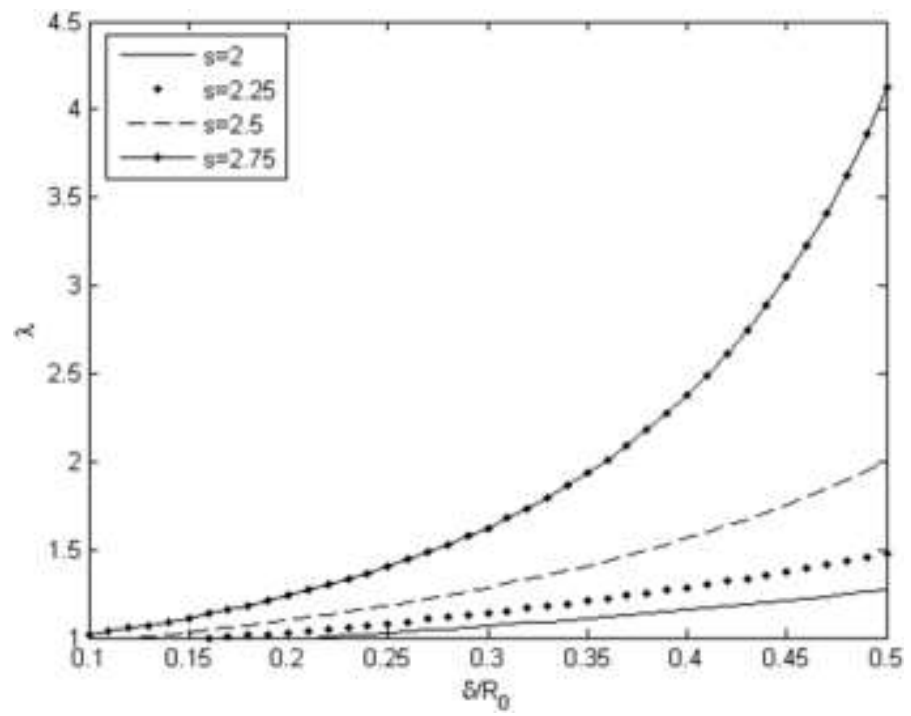

Fig 2: Variation of resistance of flow along the stenosis height for different value of stenosis shape parameter s. 


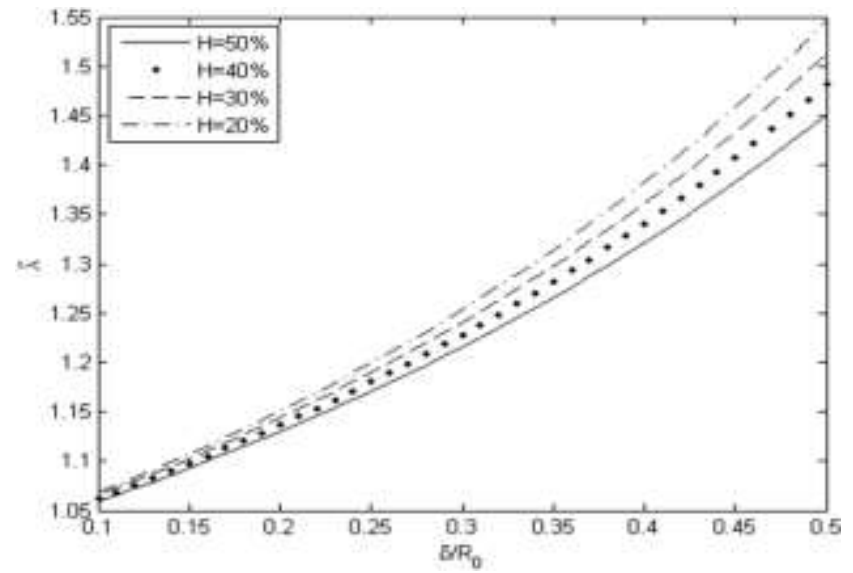

Fig 3: Variation of resistance of flow in dimensionless form along the stenosis height for different value of hematocrit level $\mathrm{H}$.

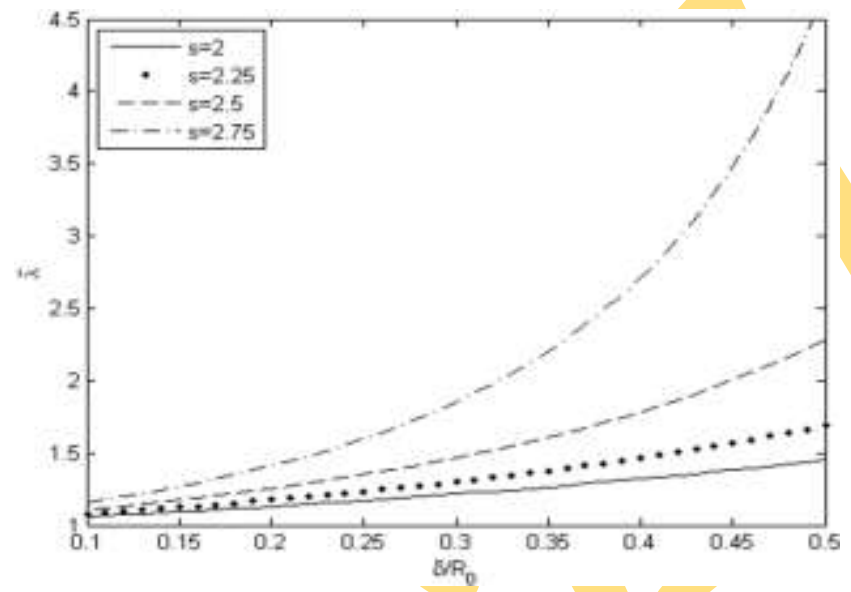

Fig 4: Variation of resistance of flow in dimensionless form along the stenosis height for different value of stenosis shape parameter s.

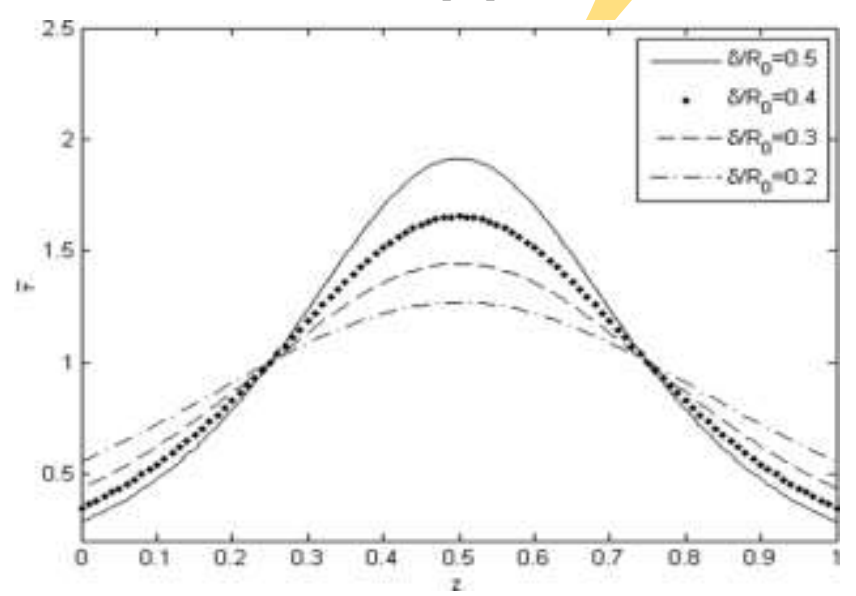

Fig 5: Variation of skin frictionin dimensionless form along $\mathrm{z}$ axis for different value of stenosis height. 


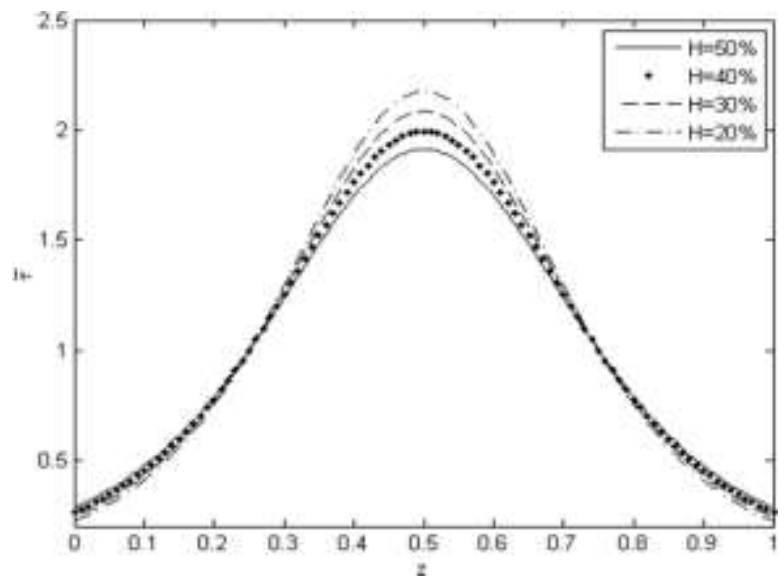

Fig 6: Variation of skin frictionin dimensionless form along $\mathrm{z}$ axis for different value of hematocrit level $\mathrm{H}$.

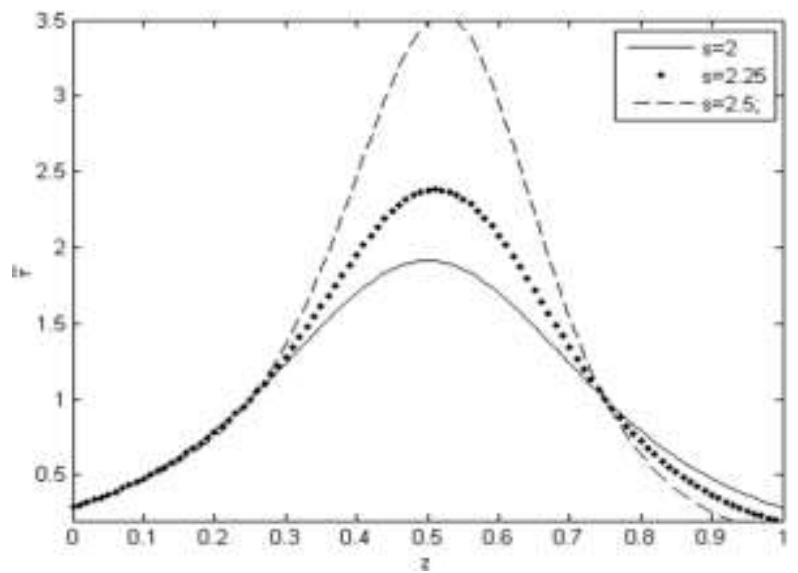

Fig 7: Variation of skin frictionin dimensionless form along $\mathrm{z}$ axis for different value of stenosis shape parameter $\mathrm{s}$.

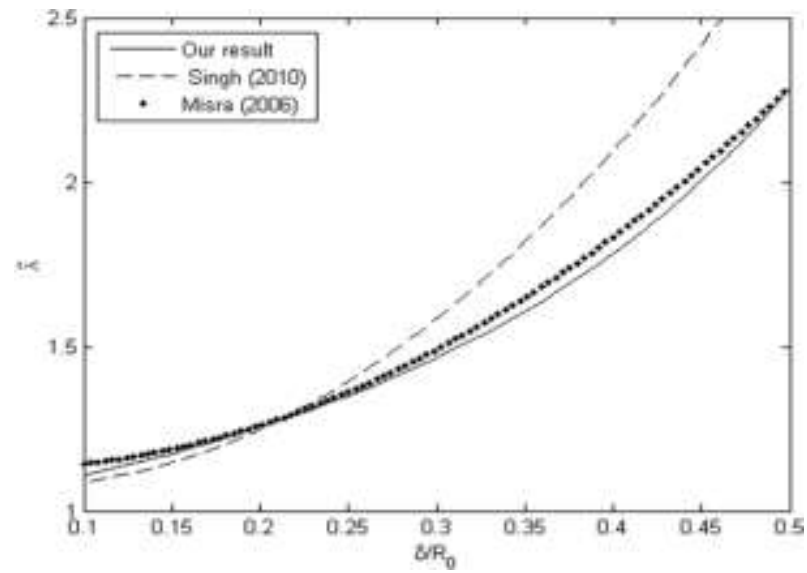

Fig.8: Comparison of resistance of our result with other researchers (Misra et.al, 2006), (B. Singh, 2010). 


\section{Conclusions}

We have focused the effect of Hematocrit on flow parameters of blood such as, resistance, frictional resistance, and skin friction due to stenosis in the artery taking blood as nonNewtonian fluid. It was found that resistance increased with the increasing of hematocrit level and stenosis height. It also showed that skin-friction increased with increasing of stenosis height and decreased with the decreasing of stenosis height. Moreover, the effect of stenosis shape parameter on resistance and skin-friction increased with the increasing of shape parameter due to different hematocrit level for different formation of stenosis shapes and similar result was observed for skin friction due to of shape parameter. Finally, we plotted the above results graphically for different parameter of flow and stenosis.

\section{REFERENCES}

B. Singh, P. Joshi and B. K. Joshi, Blood Flow through an Artery Having Radially Non-Symmetric Mild Stenosis, Applied Mathematical Sciences, Vol. 4(2010), no. 22, 1065 - 1072.

Biswas D. and Chakraborty, U.S., Pulsatile Flow of Blood in a Constricted Artery with Body Acceleration, Appl. Appl. Math., 4(2009), 329-342.

BiswasD.and U. S. Chakraborty. Impact of Hematocrit and Slip Velocity on Pulsatile Blood Flow in a Constricted Tapered Artery, International Journal of Engineering Research and Technology. 3(3), 435-449,(2010)

Blair, G. W. S., and D. C. Spanner An introduction to biorheology, Elsevier scientific publishing company, Amsterdam, Oxford and New York (1974)..

Cho, Y. I. and K. R. Kensey, Effects of the non-Newtonian viscosity of blood on hemodynamics of diseased arterial flows: part 1, steady flows, Biorheology 28(1991). 241-262.

Crowley, T. A. and V. Pizziconi), Isolation of plasma from whole blood using planar microfilters for lab-on-a-chip applications, Lab Chip 5(2005), 922-929.

Eckmann, D., Bowers S., Stecker M., Chenung A., Hematocrit volume expander, temperature and shear rate effects on blood viscosity, Anasth Analg 91(2000), 539-545.

F. J. Walburn, D. J. Schneck, A constitutive equation for whole human blood, Biorheology,13:201(1976).

Forrester J. and D. Young), Flow through a converging diverging tube and its implications in occlusive vascular disease-i, J. Biomech. 3(1970), 297-305.

Halder K., Bull. Math. Biology, 47(1985), 545-550.

Leondes, C. T., Biomechanical systems: techniques and applications, volume IV: biofluid methods in vascular and pulmonary systems: techniques and applications: 4 CRC Press (2000).

Misra, J. C. and G. C. Shit, Blood flow through arteries in a pathological state: A theoretical study, International Journal of Engineering Science, 44(2006), 662-671.

Misra, J.C. and Shit, G.C., , Role of Slip Velocity in Blood Flow through Stenosed Arteries: A NonNewtonian Model" Journal of Mechanics in Medicine and Biology, 7(2007), 337-353.

Paut O., and B. Bissonnette, Effects of temperature and haematocrit on the relationships between blood flow velocity and blood flow in a vessel of fixed diameter, British Journal of Anaesthesia, 88(2), 277-9(2002).

Pralhad R.N and D.H. Schultz, Modeling of arterial stenosis and its applications to blood diseases, Mathematical Biosciences 190(2004), 203-220.

Shukla J.B., S.P. Gupta and R.S. Rarihar, Biorheological aspects of blood flow through artery with mild stenosis: Effects of peripheral layer, Biorheology, 17(1980); 403-410.

Singh A. K. and D. P. Singh, Blood flow Obeying Casson fluid Equation through an artery with radially non-symmetric mild stenosis, American Journal of Applied Mathematics, 1(1), 1114(2012). 
SomchaiSriyab, Mathematical Analysis of Non-Newtonian Blood Flow in Stenosis Narrow Arteries, Comput Math Methods Med. 2014; 2014: 479152.

Taylor, M.G., "The Influence of Anomalous Viscosity of Blood upon its Oscillatory Flow", Physics in Medicine and Biology, 3(1959), pp. 273-290.

Ting, T. W. D. and B. S. Wu, Effects of hematocrit levels on the blood hlow in the corrugated vessel due to the implantation of intravascular stents, Biomed. Eng. Appl. Comm, 18(2), 80-86(2006).

Wong, K., J. Tu, J. Mazumdar and D. Abbott, Modelling of blood flow resistance for an atherosclerotic artery with multiple stenosis and poststenotic dilatations, Anziam J. 51 (EMAC2009), C66-C82 (2010),

Young D. F., Effect of a time - dependent stenosis on flow through a tube, J.Engg. Ind., Trans. Am. Soc. Mech. Engg. 90(1968), 248-254. 